\title{
Discovery of Thermonuclear Type-I X-ray Bursts from the X-ray binary MAXI J1807+132
}

\author{
A. C. Albayati, ${ }^{1 \star}$ D. Altamirano, ${ }^{1}$ G. K. Jaisawal, ${ }^{2}$ P. Bult,${ }^{3,4}$ S. Rapisarda, ${ }^{5}$ \\ G. C. Mancuso, ${ }^{6,7}$ T. Güver, ${ }^{8,9}$ Z. Arzoumanian, ${ }^{4}$ D. Chakrabarty, ${ }^{10}$ \\ J. Chenevez, ${ }^{2}$ J. M. C. Court, ${ }^{11}$ K. C. Gendreau, ${ }^{4}$ S. Guillot, ${ }^{12,13}$ L. Keek, ${ }^{14}$ \\ C. Malacaria, ${ }^{15,16}$ and T. E. Strohmayer ${ }^{4,17}$ \\ ${ }^{1}$ School of Physics and Astronomy, University of Southampton, Southampton, SO17 1BJ, UK \\ ${ }^{2}$ National Space Institute, Technical University of Denmark, Elektrovej 327-328, DK-2800 Lyngby, Denmark \\ ${ }^{3}$ Department of Astronomy, University of Maryland, College Park, MD 20742, USA \\ ${ }^{4}$ Astrophysics Science Division, NASA's Goddard Space Flight Center, Greenbelt, MD 20771, USA \\ ${ }^{5}$ Shanghai Astronomical Observatory, Chinese Academy of Sciences, 80 Nandan Road, Shanghai 200030 , China \\ ${ }^{6}$ Instituto Argentino de Radioastronomía (CCT-La Plata, CONICET; CICPBA), C.C. No. 5, 1894 Villa Elisa, Argentina \\ ${ }^{7}$ Facultad de Ciencias Astronómicas y Geofísicas, Universidad Nacional de La Plata, Paseo del Bosque s/n, 1900 La Plata, Argentina \\ ${ }^{8}$ Istanbul University, Science Faculty, Department of Astronomy and Space Sciences, Beyaztt, 34119, Istanbul, Turkey \\ ${ }^{9}$ Istanbul University Observatory Research and Application Center, Istanbul University 34119, Istanbul Turkey \\ ${ }^{10}$ MIT Kavli Institute for Astrophysics and Space Research, Massachusetts Institute of Technology, Cambridge, MA 02139, USA \\ ${ }^{11}$ Department of Physics and Astronomy, Texas Tech University, PO Box 41051, Lubbock, TX 79409, USA \\ ${ }^{12}$ CNRS, IRAP, 9 avenue du Colonel Roche, BP 44346, F-31028 Toulouse Cedex 4, France \\ 13 Université de Toulouse, CNES, UPS-OMP, F-31028 Toulouse, France \\ 14 cosine B.V., Oosteinde 36, 2361 HE Warmond, The Netherlands \\ ${ }^{15}$ NASA Marshall Space Flight Center, NSSTC, 320 Sparkman Drive, Huntsville, AL 35805, USA \\ ${ }^{16}$ Universities Space Research Association, Science and Technology Institute, 320 Sparkman Drive, Huntsville, AL 35805, USA \\ ${ }^{17}$ Joint Space-Science Institute, NASA's Goddard Space Flight Center, Greenbelt, MD 20771, USA
}

\begin{abstract}
MAXI J1807+132 is a low-mass X-ray binary (LMXB) first detected in outburst in 2017. Observations during the 2017 outburst did not allow for an unambiguous identification of the nature of the compact object. MAXI J1807+132 was detected in outburst again in 2019 and was monitored regularly with NICER. In this paper we report on five days of observations during which we detected three thermonuclear (Type-I) X-ray bursts, identifying the system as a neutron star LMXB. Time-resolved spectroscopy of the three Type-I bursts revealed typical characteristics expected for these phenomena. All three Type-I bursts show slow rises and long decays, indicative of mixed $\mathrm{H} / \mathrm{He}$ fuel. We find no strong evidence that any of the Type-I bursts reached the Eddington Luminosity; however, under the assumption that the brightest X-ray burst underwent photospheric radius expansion, we estimate a $<12.4 \mathrm{kpc}$ upper limit for the distance. We searched for burst oscillations during the Type-I bursts from MAXI J1807+132 and found none $(<10 \%$ amplitude upper limit at $95 \%$ confidence level). Finally, we found that the brightest Type-I burst shows a $\sim 1.6$ sec pause during the rise. This pause is similar to one recently found with NICER in a bright Type-I burst from the accreting millisecond X-ray pulsar SAX J1808.4-3658. The fact that Type-I bursts from both sources can show this type of pause suggests that the origin of the pauses is independent of the composition of the burning fuel, the peak luminosity of the Type-I bursts, or whether the NS is an X-ray pulsar.
\end{abstract}

Key words: stars: neutron - stars: individual (MAXI J1807+132) - X-rays: binaries - X-rays: bursts

* E-mail: a.c.albayati@soton.ac.uk tron star (NS) or a black hole $(\mathrm{BH})$ primary accreting ma- 
terial from a low mass $\left(\lesssim 1 \mathrm{M}_{\odot}\right)$ companion star through Roche lobe overflow. Gas flowing from the companion star forms an accretion disc around the primary. As the gas in the disc spirals closer to the compact object, gravitational potential energy is released in the form of X-rays. A LMXB spends most of its life in a quiescent (very low or non-accreting) state, but when in outburst, it can reach X-ray luminosities of $L_{\mathrm{X}} \simeq 10^{34-38} \mathrm{erg} \mathrm{s}^{-1}$ (see, e.g., Tauris \& van den Heuvel 2006, for a review).

It is challenging to differentiate between LMXBs hosting a BH or NS. Dynamical measurements of a compact object's mass can be used to help identify its nature, however, the sensitivity of the observations needed to make these estimations is difficult to acquire (e.g., Casares \& Jonker 2014). The detection of coherent pulsations confirms a NS identification, as these are associated with the spin frequency of the NS (e.g., Patruno \& Watts 2012). Similarly, thermonuclear burning requires a solid surface for the fuel to settle on. A BH has no solid surface, but an event horizon instead, hence the detection of thermonuclear burning also secures a NS identification. There are additional observables that can be used to identify the nature of the compact object, although the identification is based on empirical evidence that certain phenomenology has only been observed in either a $\mathrm{BH}$ or a NS so far. For example, quasi-periodic oscillations (QPOs) with frequencies higher than $500 \mathrm{~Hz}$ have only been seen in X-ray light curves from observations of NS systems (see, e.g., van der Klis 2006, for a review). However, the observation and characterisation of QPOs, and the power-spectral broadband noise, with frequencies below a few hundred Hertz are not always conclusive (e.g., Klein-Wolt \& van der Klis 2008). Multi-wavelength observations have been also used to distinguish between $\mathrm{BHs}$ and NSs. For example, NSs can be $\sim 30$ times fainter in the radio band when compared with BHs observed at similar X-ray luminosities. However, recent works have shown that there is a population of radio-faint $\mathrm{BHs}$ that have similar radio luminosities to NSs (Tetarenko et al. 2018).

Thermonuclear burning in a NS atmosphere can manifest as stable, unstable, or marginally stable (e.g., Galloway \& Keek 2017). Here, we concentrate on unstable thermonuclear (Type-I) X-ray bursts (hereafter referred to as "X-ray bursts"). X-ray bursts appear as a sudden increase in X-ray emission over timescales of seconds. They occur when pure or mixed material - Hydrogen, Helium, and sometimes Carbon - accreted onto the NS surface reaches a critical density and temperature which allows for runaway thermonuclear burning (see, e.g., Lewin et al. 1993; Strohmayer \& Bildsten 2003, for reviews). During this process, the X-ray flux increases rapidly $(\lesssim 1-10$ s) and is generally followed by an exponential-like decay over tens of seconds as the NS atmosphere cools.

When a Type-I X-ray burst reaches the Eddington limit, $L_{\mathrm{Edd}}$, it results in photospheric radius expansion (PRE, see, e.g., Tawara et al. 1984; Lewin et al. 1984), where the outward radiation pressure exceeds the inward gravitational force. Since black body luminosity scales as $L \propto R^{2} T^{4}$, when the X-ray burst reaches the Eddington limit an increase in radius and decrease in temperature can be seen in timeresolved spectral analysis of PRE X-ray bursts. An X-ray burst's luminosity remains roughly constant at $L_{\mathrm{Edd}}$ dur-
Table 1. Details of the NICER observations analysed in this paper. Quoted exposure times are as after processing.

\begin{tabular}{cccccc}
\hline$\#$ & ObsID & $\begin{array}{c}\text { Start Time } \\
(\text { MJD })\end{array}$ & $\begin{array}{c}\text { Date } \\
(\text { DD-MM-YY })\end{array}$ & $\begin{array}{c}\text { Exposure } \\
(\mathrm{sec})\end{array}$ & $\begin{array}{c}\text { X-ray } \\
\text { Burst }\end{array}$ \\
\hline 1 & 2200840122 & 58783.04946 & $27-10-2019$ & 15838 & - \\
2 & 2200840123 & 58784.01681 & $28-10-2019$ & 12557 & Yes \\
3 & 2200840124 & 58785.04902 & $29-10-2019$ & 5839 & Yes \\
4 & 2200840125 & 58786.27471 & $30-10-2019$ & 4480 & Yes \\
5 & 2200840126 & 58787.04898 & $31-10-2019$ & 4850 & - \\
\hline
\end{tabular}

ing PRE, thus PRE X-ray bursts can be used as empirical standard candles (van Paradijs 1978; Kuulkers et al. 2003).

MAXI J1807+132 (hereafter MAXI J1807) was first discovered by the nova-alert system of the Monitor of All-sky X-ray Image Gas Slit Camera (MAXI/GSC; Matsuoka et al. 2009; Mihara et al. 2011) during its 2017 outburst (Negoro et al. 2017). This detection was followed up with The Neil Gehrels Swift Observatory (Swift; Gehrels 2004) X-Ray Telescope (XRT; Burrows et al. 2003) observations, spectral analysis of which suggested a LMXB with a NS primary (Shidatsu et al, 2017). Further X-ray studies with XMM-Newton's European Photon Imaging Camera (EPIC; Strüder et al. 2001) and ground-based optical telescopes supported the NS identification, although the possibility of a BH primary could not be ruled out (Jiménez-Ibarra et al. 2019).

After roughly 2 years in quiescence, a new outburst of MAXI J1807 was detected on 10 September 2019 (MJD 58736) by the MAXI/GSC novaalert system (Shidatsu et al. 2019). Subsequently, the Neutron Star Interior Composition Explorer (NICER; Gendreau \& Arzoumanian 2017) monitored the source from 16 September (MJD 58742), and observed the system on a regular basis. The outburst of MAXI J1807 was characterised by flaring events lasting days to weeks (Rapisarda et al. 2019). Two X-ray bursts were detected by NICER on 28 and 29 October, and preliminary analysis of these two events reported by Arzoumanian et al. (2019) confirmed MAXI J1807 as a neutron star LMXB. In this paper we report on the detection of a third X-ray burst with NICER, and present a detailed analysis of all three Type-I bursts.

\section{OBSERVATIONS AND DATA ANALYSIS}

$N I C E R$ is an X-ray telescope on board the International Space Station. It was launched in 2017 with instrumentation specifically designed for the study of NSs. NICER's X-ray Timing Instrument (XTI) operates in the $0.2-12 \mathrm{keV}$ energy band, providing high timing and spectral resolution of $100 \mathrm{~ns}$ and $6<E / \Delta E<80$ from $0.5 \mathrm{keV}$ to $8 \mathrm{keV}$ respectively. With 52 active detectors, NICER provides an effective area of $1900 \mathrm{~cm}^{2}$ at $1.5 \mathrm{keV}$.

NICER observed MAXI J1807 between 16 September and 26 November 2019, generating a total of 47 observation IDs (ObsIDs). We searched all available data for X-ray bursts; here we report on the five observations around the time of the detection of three X-ray bursts (see Table 1). The observations correspond to ObsIDs 2200840122-26, per- 


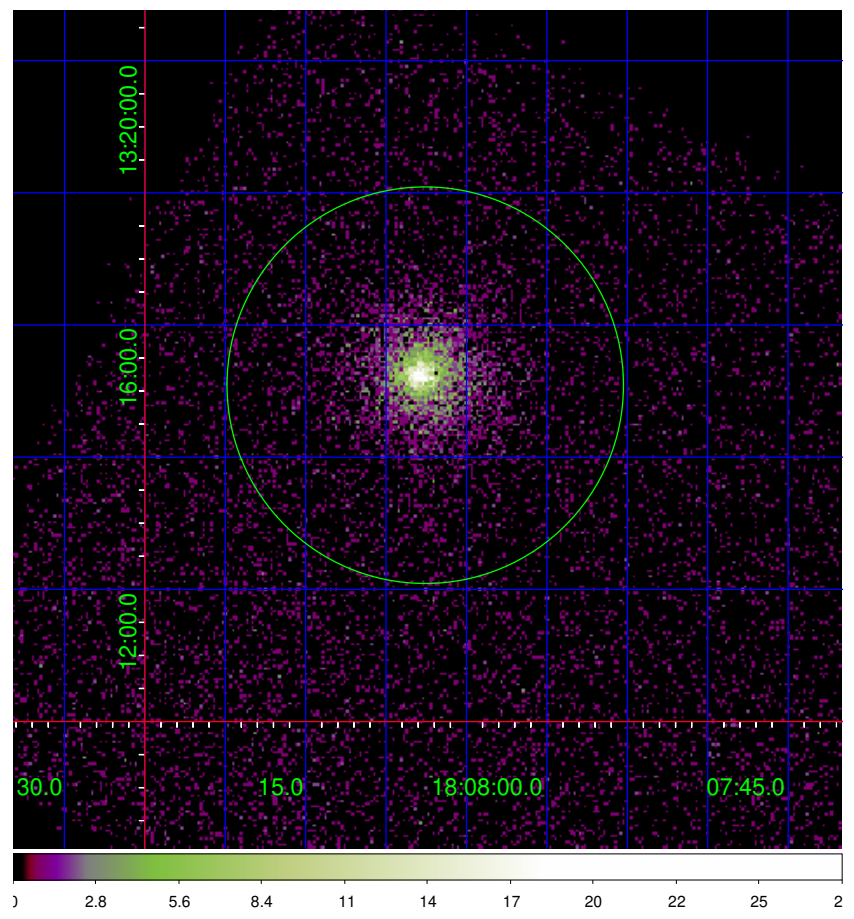

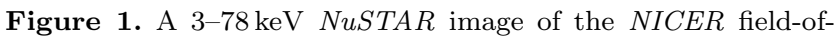
view, 26 September 2019 (ObsID 90501342002, exposure time of $22 \mathrm{ks}$ ). The NICER field-of-view for our observations is denoted by a green circle of 3 arcmin radius.

formed between 27 October (MJD 58783) and 31 October (MJD 58787), where X-ray bursts are observed in ObsIDs 2200840123-25. The detailed analysis of the spectral and variability evolution of the full outburst will be presented elsewhere.

To process the data, we used HEASOFT v6.26.1 and NICERDAS v6 (HEASARC 2014), and applied standard filtering criteria, i.e., pointing offset $<54^{\prime \prime}$, Earth limb elevation angle $>15^{\circ}$, and bright Earth limb angle $>30^{\circ}$. Because the observations occurred during an epoch of high optical loading, the parameter "underonly_range" was increased to $0-400$ when reprocessing the data using the nicer12 tool. The first X-ray burst occurred during a nominal South Atlantic Anomaly (SAA) passage. As such, "nicersaafilt" was set to "no" on the second observation analysed, in order to include the data taken during the SAA passage. To test if the data were affected by the apparent passage through the SAA, we extracted the $13-15 \mathrm{keV}$ lightcurve to look for the presence of high-energy background flares (see Bult et al. 2018); we found none. The total good exposure after processing was $43.6 \mathrm{ksec}$.

We determined the background contribution of our observations from NICER observations of a Rossi X-ray Timing Explorer (RXTE; Bradt et al. 1993; Jahoda et al. 2006) blank-sky region $\left(\tau 1-2\right.$ cts s $^{-1}$ from $\left.R X T E-6\right)$.

\subsection{Light Curves and Hardness Ratios}

To study the X-ray bursts in the context of MAXI J1807's outburst, we constructed a $0.3-10 \mathrm{keV}$ long-term light curve using $25 \mathrm{~s}$ bins. We extracted individual X-ray burst light curves in the $0.3-10 \mathrm{keV}$ energy band using $0.1 \mathrm{~s}$ bins.
Table 2. The black body temperatures, photon indices, 0.5$10 \mathrm{keV}$ unabsorbed fluxes, and reduced $\chi^{2}$ obtained from modelling the pre-burst emissions of MAXI J1807, with all errors quoted at a $90 \%$ confidence interval.

\begin{tabular}{ccccc}
\hline Burst & $\begin{array}{c}k T_{b b} \\
(\mathrm{keV})\end{array}$ & $\begin{array}{c}\text { Photon } \\
\text { Index }\end{array}$ & $\begin{array}{c}\text { Unabsorbed Flux } \\
\left(10^{-10} \mathrm{erg} \mathrm{cm}^{-2} \mathrm{~s}^{-1}\right)\end{array}$ & $\begin{array}{c}\chi_{\nu}^{2} \\
\left(\chi^{2} / \mathrm{dof}\right)\end{array}$ \\
\hline 1 & $0.10 \pm 0.01$ & $2.1 \pm 0.2$ & $0.54 \pm 0.03$ & $205.8 / 177$ \\
2 & $0.19 \pm 0.01$ & $2.0 \pm 0.2$ & $1.75 \pm 0.05$ & $263.2 / 233$ \\
3 & $0.11 \pm 0.02$ & $2.6 \pm 0.3$ & $1.14 \pm 0.04$ & $214.8 / 178$ \\
\hline
\end{tabular}

We define the start of each X-ray burst as the first $0.1 \mathrm{~s}$ bin which has an intensity $1 \sigma$ above the persistent emission (calculated as the median count rate of the exposure) when scanning backwards in time from the X-ray burst peak. We define the end of an X-ray burst as the initial time of a $10 \mathrm{~s}$ bin that is consistent within $1 \sigma$ of the median count rate of the last $100 \mathrm{~s}$ of the data segment containing the X-ray burst.

The hardness ratio was defined as the count rate in the $1-10 \mathrm{keV}$ energy band divided by the count rate in the 0.3 $1 \mathrm{keV}$ energy band. Light curves for the hardness ratios were extracted with $0.2 \mathrm{~s}$ binning.

\subsection{Spectral Analysis}

\subsubsection{Persistent Emission}

Before examining the $\mathrm{X}$-ray burst emissions, the pre-burst (persistent) spectra of MAXI J1807 were first explored. For this, we extracted the spectra of the persistent emission from exposures of $509 \mathrm{~s}, 395 \mathrm{~s}$, and $110 \mathrm{~s}$ before the first, second, and third X-ray bursts, respectively. The $0.5-10 \mathrm{keV}$ energy spectrum was then successfully fitted with an absorbed black body plus power-law model in Xspec v12.10.1:

tbabs (bbodyrad + powerlaw),

where tbabs takes into account the effect of the interstellar absorption (Wilms et al. 2000). This simple model fits the data well. As the burst flux is generally much brighter than the persistent emission, our results remain unaffected if we use more complex models for the persistent emission. We found a column density of $N_{H}=(1.3 \pm 0.9) \times 10^{21} \mathrm{~cm}^{-2}$ before the first X-ray burst and chose the same value to fit the persistent emission spectra before the second and third $\mathrm{X}$-ray bursts. We note that our results were the same within error by (i) using the column density as explained above, (ii) using column densities as estimated from the persistent emission before each burst, or (iii) using an average of the three column densities.

The black body temperatures, photon indices, $0.5^{-}$ $10 \mathrm{keV}$ unabsorbed fluxes, and reduced $\chi^{2}$ obtained from modelling the pre-burst emissions are reported in Table 2 . In all cases, we obtained a reduced $\chi^{2}$ of $\sim 1.1$. The errors are quoted for a $90 \%$ confidence interval.

\subsubsection{Time-Resolved Spectroscopy}

We performed time-resolved spectroscopy on all three X-ray bursts. Each X-ray burst was divided into time bins containing a minimum of 500 counts. We fitted energy spectra 
to each time bin using the variable persistent flux method ( $f_{a}$-method; see, e.g., Worpel et al. 2013, 2015).

The $f_{a}$-method describes the X-ray burst thermal emission with a black body component and any possible excess is accounted for by scaling the pre-burst emission. This model is usually constructed by first fixing the pre-burst spectral components and then adding a black body component for the X-ray burst emission. A multiplicative factor $f_{a}$ is then applied on the persistent part, which allows us to account for the effect of X-ray burst emission on the accretion processes. In Xspec, this model reads as:

tbabs ( bbodyrad $+f_{a}$ (bbodyrad + powerlaw) ),

where tbabs takes into account the interstellar absorption and bbodyrad the black body emission from the X-ray burst. In our study, the model for the persistent emission (bbodyrad + powerlaw) is fixed to the respective values we found in Section 2.2.1.

The $f_{a}$-method has already been used for time-resolved spectroscopy of X-ray bursts using NICER data (see, e.g., Keek et al. 2018; Jaisawal et al. 2019). Although the $f_{a}$ method has been shown to be a good way to parameterise the effects on the accretion disc, it is likely that $f_{a}$ should be a function of energy rather than a constant (see, e.g., Keek et al. 2014; Degenaar et al. 2018). In our analysis, given the relatively low count rates during MAXI J1807's X-ray bursts, we assumed an $f_{a}$ that does not depend on energy.

\section{RESULTS AND DISCUSSION}

\subsection{X-ray Imaging Observations}

NICER is a non-imaging instrument. Here we investigate whether the X-ray bursts could originate from a different source than MAXI J1807.

MAXI J1807 was observed with NuSTAR (Harrison et al. 2013) on 26 September 2019, i.e., about 35 days before the first X-ray burst we detected with NICER. In Figure 1 we show the $3-78 \mathrm{keV} N U S T A R$ image including the NICER field-of-view (a circle with 3 arcmin radius, corresponding to a 30 arcmin $^{2}$ field-of-view; Gendreau \& Arzoumanian 2017). Only one source was significantly detected, with coordinates consistent with that of MAXI J1807.

There are also 28 photon counting (PC) mode Swift/XRT observations taken over the period 26 March30 May 2017 (there are no Swift/XRT imaging observations during 2019). We created a $0,3-10 \mathrm{keV}$ image of the NICER field-of-view by integrating the 28 images (total exposure time of $24 \mathrm{ks}$ ). The image was reduced using the Swift/XRT data product generator provided by the University of Leices$\operatorname{ter}^{1}$. Only one source was significantly detected, with coordinates consistent with that of MAXI J1807. Given the evidence provided by NuSTAR and Swift/XRT, we concluded that the X-ray bursts originate from MAXI J1807.

1 https://www.swift.ac.uk/user_objects/index.php

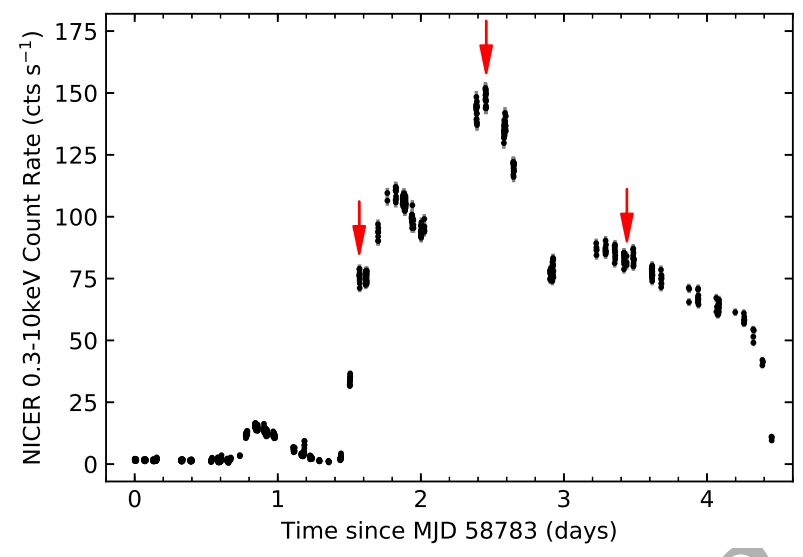

Figure 2. NICER long-term light curve of MAXI J1807 with $25 \mathrm{~s}$ time resolution in the $0.3-10 \mathrm{keV}$ energy band. This section of the outburst contains the three thermonuclear X-ray bursts detected in October 2019. The X-ray burst data have been removed for clarity, and their onsets marked by arrows.

\subsection{Outburst Evolution and Occurrence of X-ray bursts}

Figure 2 shows the outburst evolution of MAXI J1807 from 27 to 31 October 2019 (MJD 58783-58787). The occurrence of the X-ray bursts are marked by arrows. On 27 October $(t \approx 0.7$ days $)$, the light curve shows a flare-like feature, where the count rate rises from $\sim 2 \mathrm{cts} \mathrm{s}^{-1}$ to $\sim 15 \mathrm{cts} \mathrm{s}^{-1}$ and falls back to the original count rate within half a day. Roughly 3 hrs later, we observe the onset of a larger flare with multiple peaks. The count rate increases to $\sim 70 \mathrm{cts} \mathrm{s}^{-1}$ over approximately $3 \mathrm{hrs}$, after which the first X-ray burst occurs on MJD $58784.61727(t \approx 1.6$ days in Figure 2$)$. The flare reaches a first peak at $\sim 110 \mathrm{cts} \mathrm{s}^{-1}$ roughly $4.5 \mathrm{hrs}$ after the X-ray burst, and drops to $\sim 95 \mathrm{cts} \mathrm{s}^{-1}$ in the following $4 \mathrm{hrs}$. There is an additional peak almost $10 \mathrm{hrs}$ later during which the second X-ray burst occurs on MJD $58785.50558(t \approx 2.5$ days in Figure 2$)$ at a persistent count rate of $\sim 150 \mathrm{cts} \mathrm{s}^{-1}$. The persistent count rate then drops to $\sim 80 \mathrm{cts} \mathrm{s}^{-1}$ over approximately $10 \mathrm{hrs}$ before a final rise to $\sim 90 \mathrm{cts} \mathrm{s}^{-1}$, after which the flare begins to decay. At this stage in the outburst the third X-ray burst occurs on MJD $58786.48803(t \approx 3.5$ days in Figure 2$)$ at a persistent count rate of $\sim 80 \mathrm{cts} \mathrm{s}^{-1}$. There is a sudden decrease in flux just less than a day after the third X-ray burst, where we observe the flux decreasing from $\sim 60 \mathrm{ctss}^{-1}$ to $\sim 10 \mathrm{ctss}^{-1}$ in roughly $4 \mathrm{hrs}$.

We observed the three X-ray bursts over a period of three days. The waiting time between the first and second $\mathrm{X}$-ray bursts was $21.3 \mathrm{hrs}$, while between the second and third X-ray bursts was $23.6 \mathrm{hrs}$. We note that the data-gaps prevent us to understand whether there were other X-ray bursts than those we detected.

\subsection{Type-I X-ray Burst Light Curves}

Figure 3 shows light curves and hardness ratios of the three X-ray bursts exhibited by MAXI J1807. The first, second, and third X-ray bursts will hereafter be referred to as B1, $\mathrm{B} 2$, and $\mathrm{B} 3$, respectively. At the burst onset, $t_{B 1}=0, \mathrm{~B} 1$ count rate increases from the persistent rate of $\sim 62 \mathrm{cts} \mathrm{s}^{-1}$ 

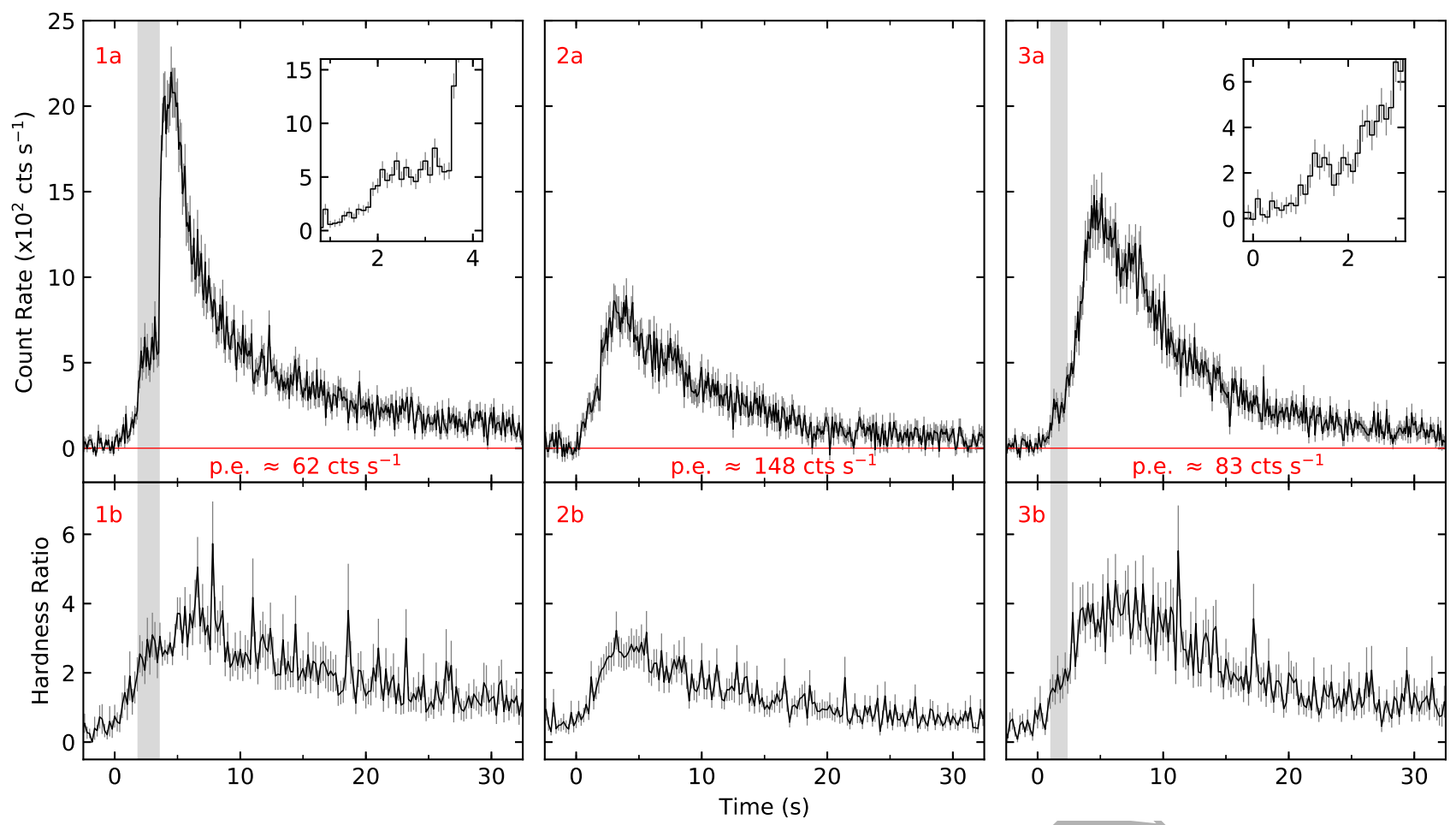

Figure 3. Top panels: light curves of the three X-ray bursts from MAXI J1807 with $0.1 \mathrm{~s}$ time resolution in the $0.3-10 \mathrm{keV}$ energy band. We found the persistent emission levels by taking the mean count rate for $100 \mathrm{~s}$ of data before the start of each X-ray burst, and subtracted them from the light curves. The persistent emission count rates are denoted on the panels as "p.e.". The insets in panels 1a and 3a show rise features in detail, which are also shaded in the main panels. Bottom panels: hardness ratios $(1-10 \mathrm{keV} / 0.3-1 \mathrm{keV}$ count rate) with $0.2 \mathrm{~s}$ time resolution.

to $\sim 500 \mathrm{cts} \mathrm{s}^{-1}$ over approximately $2 \mathrm{~s}$. At this point, the burst exhibits a "pause" lasting $\sim 1.6 \mathrm{~s}$ (shaded region and inset in Figure 3, panel 1a). Following the pause, the burst reaches its peak in roughly $0.2 \mathrm{~s}$. The count rate remains approximately constant during this peak at $\sim 2250 \mathrm{cts} \mathrm{s}^{-1}$ between $t_{B 1}=3.8-5.1 \mathrm{~s}$. After the peak, the count rate decreases for roughly $133 \mathrm{~s}$ to a persistent rate of $\sim 70 \mathrm{cts} \mathrm{s}^{-1}$, i.e., at a flux $\sim 13 \%$ higher than the persistent emission before the burst onset. The overall burst duration is $\sim 137 \mathrm{~s}$.

The rise of $\mathrm{B} 2$ is in two parts. In the initial rise, starting at $t_{B 2}=0$, the count rate increases from the persistent rate of $\sim 148 \mathrm{cts} \mathrm{s}^{-1}$ to $\sim 460 \mathrm{cts} \mathrm{s}^{-1}$ over ápproximately $2 \mathrm{~s}$. The count rate then rises suddenly and reaches the peak of the burst in less than one second. This peak has an average count rate of $\sim 920 \mathrm{cts} \mathrm{s}^{-1}$ between $t_{B 2}=2.6-4.6 \mathrm{~s}$. As the data segment containing the burst ends less than $100 \mathrm{~s}$ after the burst peak, the end of the burst is ill-defined while the count rate is still $8 \%$ above the pre-burst level $\left(160 \mathrm{cts} \mathrm{s}^{-1}\right.$, the median count rate of the last $20 \mathrm{~s}$ of the data segment containing B2).

B3 exhibits interesting features in both its rise and decay. At $t_{B 3}=0$, B3's count rate increases for roughly $1.5 \mathrm{~s}$ from the persistent rate of $\sim 83 \mathrm{cts} \mathrm{s}^{-1}$ to a potential pause similar to that we observed in B1, but at $\sim 290 \mathrm{cts} \mathrm{s}^{-1}$ (marked by the shaded region and inset in Figure 3, panel 3a). After the potential pause, the count rate continues to rise reaching a peak average count rate of $\sim 1417 \mathrm{cts} \mathrm{s}^{-1}$ between $t_{B 3}=4.0-5.3 \mathrm{~s}$. During the decay there is indication of a double peak at $t \approx 7.5 \mathrm{~s}$ lasting roughly $2 \mathrm{~s}$; how- ever the double peak is not statistically significant given the large error bars. After this, the count rate decreases over approximately $78 \mathrm{~s}$ to a post-burst persistent count rate of $\sim 100 \mathrm{cts} \mathrm{s}^{-1}$, which is $\sim 20 \%$ higher than the pre-burst persistent rate. The overall burst duration is $\sim 83 \mathrm{~s}$.

The hardness ratios of all three X-ray bursts track similar profiles to the light curves, increasing through the burst rise and decreasing through the decay. The hard band (1$10 \mathrm{keV}$ ) dominates after less than $1 \mathrm{~s}$ in each case, and increases to peaks of $\sim 4$ in B1 and B3, and $\sim 3$ in B2. There is a plateau in the hardness ratio that starts during the pause in B1 and appears to continue after it. There is also evidence of a plateau during the potential pause found in B3, after which the ratio increases; however in this case the plateau is not as well constrained as in B1.

We measure different average count rates for the persistent emission before and after each X-ray burst. Given our data set, we are unable to understand if this is due to an intrinsic change in the flux of the persistent emission, or whether it is the effect of long decay tails which we cannot differentiate from the continuum (see, e.g., in't Zand et al. 2017). Out of the three X-ray bursts, B1 has the highest peak count rate, whilst B2 has the lowest. All three X-ray bursts have a rise time of $\sim 4 \mathrm{~s}$ and exhibit long decay tails ( $>1$ minute). A slow rise and long decay is indicative of H-rich fuel at the moment of ignition, which is likely the result of accretion of a mixed H/He fuel (Galloway et al. 2008; Galloway \& Keek 2017; Schatz et al. 2001). 


\subsection{X-ray Burst Spectral Characteristics}

In Figure 4 we show the best-fit parameters for the timeresolved spectroscopy of the X-ray bursts. The bolometric unabsorbed flux $\left(F_{\mathrm{bol}}\right)$ of all three X-ray bursts approximately follows the light curve contours. The peak fluxes are reported in Table 3 . The black body temperature $\left(k T_{b b}\right)$ also follows the contours of the burst profiles in the light curves, increasing during the burst rise and decreasing during the decay. The evolution of $k T_{b b}$ is typical of the heating and cooling processes of the NS atmosphere during a Type-I Xray burst (see, e.g., Lewin et al. 1993, for a review), thus strongly suggesting that these features are indeed thermonuclear in origin. The peak temperatures achieved by each of MAXI J1807's X-ray bursts ( $\sim 1 \mathrm{keV}$, reported in Table 3) are lower than what has typically been seen in previous works for other sources $(\gtrsim 2 \mathrm{keV})$. We did not find any evidence that the low temperatures are due to any instrumental effects or due to our spectral modelling. Indeed, previous results on other sources using NICER data and the $f_{a}$-method have shown that X-ray bursts can reach temperatures in the $2-3 \mathrm{keV}$ range (see, e.g., Keek et al. 2018). Therefore, we conclude that the low temperatures we measure are intrinsic to the X-ray bursts detected in this source.

Thermonuclear X-ray bursts can be used to estimate the distance to the system if they exhibit photospheric radius expansion. The brightest of the three X-ray bursts, B1, does not show strong evidence, if any, of PRE. However, under the assumption that B1 reached the Eddington luminosity $\left(L_{\mathrm{Edd}}\right)$, MAXI J1807 contains a $1.4 M_{\odot} \mathrm{NS}$ and $L_{\mathrm{Edd}}=2 \times 10^{38} \mathrm{erg} \mathrm{s}^{-1}$ (as expected for H-rich fuel, see, e.g., Lewin et al. 1993), we found a distance upper limit of $12.4 \mathrm{kpc}$.

We see differences in the evolution of the black body radius, calculated assuming a distance of $12.4 \mathrm{kpc}$, during the three X-ray bursts. For B1, it remains approximately constant at $\sim 28.5 \mathrm{~km}$. The radius during B2 approximately follows the contour of the light curve, peaking at $\sim 28.4 \mathrm{~km}$. In the case of B3, the radius increases throughout the rise in count rate, and then remains roughly constant at $\sim 28.5 \mathrm{~km}$ after the burst peak. The radii reported here are larger than what might be expected (see, e.g., Galloway et al. 2008). This is due to the fact that they are calculated using the $12.4 \mathrm{kpc}$ upper limit for the distance. If MAXI J1807 were at, for example, half this distance, the peak radius would be roughly $18.5 \mathrm{~km}$, which, whilst it is still large, is more consistent with the expected radius during X-ray bursts.

The parameter $f_{a}$ describes the photospheric evolution of the X-ray burst as well as its effect on the accretion processes. For B2, $f_{a}$ remains at $\sim 1.9$ throughout the burst. For B1 and B3, $f_{a}$ increases and peaks at the same time as the burst, and decreases afterwards. This can be the result of real changes in the aecretion disc or due to the choice of model. Testing whether the accretion disc varies significantly is difficult to assess (e.g., Degenaar et al. 2018), particularly with the low count rates in our data.

In Table 3, we report the X-ray burst fluences and time seales $\tau\left(=\right.$ fluence / peak $\left.F_{b o l}\right)$. We calculated the fluences of B1, B2, and B3 over the first $31 \mathrm{~s}, 25 \mathrm{~s}$, and $42 \mathrm{~s}$ of data, respectively (during these time-intervals the fluence was well constrainted). The fluences are in the $(\sim 2$ to $\sim 3.5) \times 10^{-8}$ $\mathrm{erg} \mathrm{cm} \mathrm{cm}^{-2}$ range, which is the lower end of typical fluence
Table 3. The best fit parameters obtained from time-resolved spectroscopy for peak black body temperatures and bolometric fluxes achieved by each X-ray burst from MAXI J1807, and calculated estimates for X-ray burst parameters fluence and $\tau$.

\begin{tabular}{ccccc}
\hline $\begin{array}{c}\text { Burst Peak } k T_{b b} \\
(\mathrm{keV})\end{array}$ & $\begin{array}{c}\text { Peak } F_{\mathrm{bol}} \\
\left(10^{-8} \mathrm{erg} \mathrm{s}^{-1} \mathrm{~cm}^{-2}\right)\end{array}$ & $\begin{array}{c}\text { Fluence } \\
\left(10^{-8} \mathrm{erg} \mathrm{cm}^{-2}\right)\end{array}$ & $\begin{array}{c}\tau \\
(\mathrm{s})\end{array}$ \\
\hline 1 & $1.21_{-0.12}^{+0.14}$ & $1.01_{-0.15}^{+0.17}$ & $2.30 \pm 0.29$ & $2.29 \pm 0.07$ \\
2 & $0.9 \pm 0.1$ & $0.26 \pm 0.03$ & $1.36 \pm 0.11$ & $5.30 \pm 0.13$ \\
3 & $0.9 \pm 0.07$ & $0.56_{-0.05}^{+0.06}$ & $3.17 \pm 0.27$ & $5.70 \pm 0.06$ \\
\hline
\end{tabular}

distributions found in other sources (Galloway et al. 2008; Lyu et al. 2015; Boirin et al. 2007). However, given the uncertainties in our calculations of X-ray burst duration and the intensity of the continuum after the burst, our fluences are probably are underestimated by $10-15 \%$. We found that $\tau$ was in the $\sim 2$ to $\sim 6$ s range. These values are also in the lower end of the distribution usually found in other sources Galloway et al. (2008, 2020).

Calculating an accurate value for the X-ray burst parameter $\alpha$ (ratio of the integrated persistent flux to the burst fluence, see, e.g., Galloway et al. 2008) is not possible without a well-constrained recurrence time. However, as an exercise, we calculate a rough estimate for $\alpha$ with certain caveats. We only detected X-ray bursts from MAXI J1807's 2019 outburst during a $\sim 1.9$ day window. During this window, NICER covered $\sim 8 \%$ of the time. If we assume that only $\sim 8 \%$ of the X-ray bursts were detected in this time frame, this implies a total of $\sim 36$ X-ray bursts with a recurrence time of $\sim 1.27 \mathrm{hrs}$. Taking a pre-burst flux of $\sim 1 \times 10^{-10} \mathrm{erg} \mathrm{s}^{-1} \mathrm{~cm}^{-2}$ and fluence of $\sim 2 \times 10^{-8} \mathrm{erg} \mathrm{cm}^{-2}$, this gives an averaged $\alpha \approx 23$. These assumptions make the statistical and systematic uncertainties on this calculation rather large. Comparing this value with what we see in Galloway et al. (2008), our estimate for $\alpha$ is much smaller than what we would expect for our calculated values of $\tau$ (if compared with other sources, we expect $\alpha>60$ for $\tau<10$; see Figure 14, Galloway et al. 2008). The uncertainty in our calculations may be able to account for this tension, although it may also be an intrinsic property of the X-ray bursts in MAXI J1807 as the fluences we measured are smaller than average.

\subsection{Search for Burst Oscillations}

To search for burst oscillations, we constructed $0.8-8.0 \mathrm{keV}$ $\mathrm{X}$-ray burst light curves at 1/8192 s time resolution. Each light curve started $10 \mathrm{~s}$ prior to the burst onset, and had a duration of $50 \mathrm{~s}$, such that the entire burst epoch was included. We then applied a $T=2 \mathrm{~s}, 4 \mathrm{~s}$, and $8 \mathrm{~s}$ wide window to each light curve, which we moved across the respective burst in steps of $T / 2$. For each combination of window size and position we constructed the power spectrum and searched between $100 \mathrm{~Hz}$ and $1000 \mathrm{~Hz}$ for the presence of a coherent burst oscillation signal in excess of the counting noise distribution (see Watts et al. 2005; and Watts 2012 for a review). No significant signals were observed to a $95 \%$ confidence upper limit of approximately $10 \%$ fractional amplitude at peak intensity. 

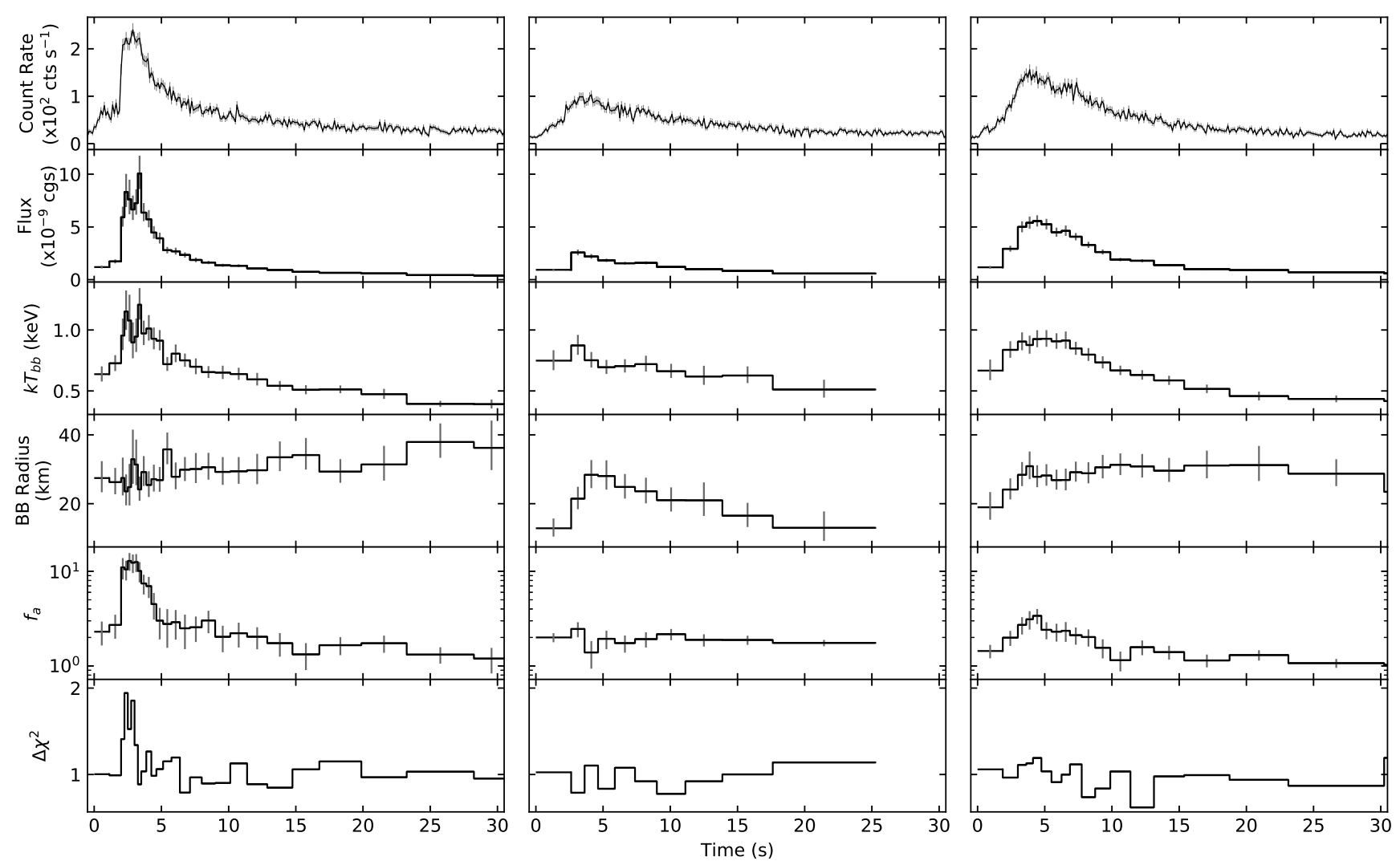

Figure 4. Time-resolved spectroscopy of the three X-ray bursts (B1, B2 and B3, left to right, respectively) using the variable $f_{a}$ method. From top, we show the count rate, the estimated bolometric flux (in units of $\mathrm{erg} \mathrm{s}^{-1} \mathrm{~cm}^{-2}$ ), the black body temperature and radius (assuming a distance of $12.4 \mathrm{kpc}$ ), the variable scale factor $f_{a}$ and the reduced $\chi^{2}$.

\subsection{Pauses and Comparison}

Historically, the rises of thermonuclear Type-I X-ray bursts have generally been described as relatively fast and smooth. In the last two decades, thanks to the high time resolution and effective area of $R X T E$, we have learned that $\mathrm{X}$-ray burst profiles can be very complex. These include slower or faster rises, two-step rises (like we see in panel $2 \mathrm{a}$ of Fig 3), concave and convex rises (Maurer \& Watts 2008), and X-ray bursts with multiple (2 or 3) peaks. We note that, sometimes an X-ray burst with a double-peaked profile can be interpreted as two different events (e.g., Bhattacharyya \& Strohmayer 2007).

X-ray bursts with multiple peaks in their bolometric luminosity profile are not uncommon (see, e.g., Galloway et al. 2008; Lewin et al. 1993; Watts \& Maurer 2007, and references therein). Notably, the source $4 \mathrm{U} 1636-536$ shows the largest sample of multi-peaked X-ray bursts with a variety of profiles, including X-ray bursts with two peaks that reach the samé luminosity (e.g., Bhattacharyya \& Strohmayer 2006a), X-ray bursts where the two peaks are not at the same luminosity (e.g., Bhattacharyya \& Strohmayer 2006b; Maurer \& Watts 2008), and even triple-peaked X-ray bursts (van Paradijs et al. 1986; Zhang et al. 2009). Double-peaked $\mathrm{X}$-ray bursts from this source have been interpreted in a number of different ways. For example, a second peak may be caused by multi-step thermonuclear energy release, or the ignition of fresh or leftover material (see, e.g., Jaisawal et al. 2019, and references therein). Bhattacharyya \& Strohmayer (2006a,b) interpreted the double-peaked profile as the result of a temporary stalling in the burning front at the NS's equator. This interpretation requires rare high-latitude ignition (Spitkovsky et al. 2002), explaining why doublepeaked bursts are far less common than single-peaked bursts (e.g., Galloway et al. 2008, but also see discussions by Cooper \& Narayan (2007); Watts \& Maurer (2007); Maurer \& Watts (2008)). However, triple-peaked bursts are difficult to explain solely by a temporary stalling in the burning front. Zhang et al. (2009) showed that observations of triple peaks pose particular challenges for the polar ignition mechanism required by the interpretation of Bhattacharyya \& Strohmayer (2006a,b).

In this paper, we report on the discovery of a $<2 \mathrm{sec}$ pause in the rise of one single-peaked burst in the LMXB MAXI J1807+132. Given the complex profiles that X-ray bursts may have (see above), it is important to understand whether pauses during an X-ray burst rise are a different manifestation of the multi-peak phenomena (i.e. what we observe as a pause is in reality a very short, weak peak of a double-peaked X-ray burst), or the pause is unrelated to the physical process that produces multi-peak X-ray bursts.

Bult et al. (2019) recently reported on a bright, Hefueled PRE Type-I X-ray burst from the accreting millisecond X-ray pulsar SAX J1808.4-3658. The light curve profile 
of the X-ray burst (hereafter S1) was complex: it showed a pause in the rise and a double peak unrelated to the PRE event. Additionally, S1 showed burst oscillations. Bult et al. (2019) found that the pause, the dip between the X-ray burst's double peaks, and the onset of burst oscillations all occur at similar count rates, suggesting that the similar count rates are related to the Eddington limit of a hydrogen envelope.

The pause in the first burst we detected in MAXI J1807 (B1) is $\sim 1.6 \mathrm{~s}$ in duration, and occurs at roughly $20 \%$ of the peak count rate. The pause in SAX J1808.4-3658's S1 is shorter, lasting $\sim 0.7 \mathrm{~s}$, and occurs at roughly $40 \%$ of the peak count rate. We calculated the ratio between peak and pause fluxes to be $5.8_{-0.2}^{+0.3}$, whereas this ratio was calculated to be $1.68 \pm 0.13$ for $\mathrm{S} 1$. In both $\mathrm{B} 1$ and $\mathrm{S} 1$, the rise time before the pause is roughly the same duration as the pause, lasting $\sim 1.6 \mathrm{~s}$ and $\sim 0.6 \mathrm{~s}$ for B1 and S1 respectively. However, the rise time from the end of the pause to the burst peak in $\mathrm{B} 1$ is $\sim 0.5 \mathrm{~s}$, much shorter than that of $\sim 3 \mathrm{~s}$ in $\mathrm{S} 1$. $\mathrm{B} 1$ and $\mathrm{S} 1$ also have different fuel compositions, with mixed $\mathrm{H} / \mathrm{He}$ (H-rich) and He only, respectively.

Although we currently have a sample of only two Xray bursts with confirmed pauses during the rise, we suggest that the pauses are not a shorter and weaker manifestation of the multi-peaked X-ray bursts seen in other sources. Our interpretation is supported by the fact that SAX J1808.43658's X-ray burst (Bult et al. 2019) exhibits both a pause and a double-peaked profile, showing that the two phenomena can manifest in the same X-ray burst. In addition, the X-ray burst we report for MAXI J1807 shows a pause and a single-peaked profile. These two results together, and the lack of other reports on pauses in X-ray bursts studied with $R X T E$, suggest that there is no link between the detection of double-peaked profiles and the detection of a pause during the rise. However, our interpretation would be incorrect if the pauses are short and weak manifestations of a peak in the X-ray burst profile, meaning that the X-ray burst in SAX J1808.4-3658 can be considered a triple-peaked X-ray burst, and B1 in MAXI J1807 can be considered a doublepeaked burst.

in't Zand et al. (2003) reported on an unusual TypeI X-ray burst from GRS $1747-312^{2}$ observed with $R X T E$ whilst the system was apparently in quiescence. The 2$10 \mathrm{keV}$ light curve profile exhibits a pause-like feature in the rise of the X-ray burst, while the full $2-60 \mathrm{keV}$ light curve did not show a pause. The pause in the $2-10 \mathrm{keV}$ light curve is considerably longer than those seen in S1 and B1 $(\sim 20 \mathrm{~s})$, and, judging from Figure 2a of in't Zand et al. (2003), the pause occurs at roughly $70 \%$ of the peak count rate. The X-ray burst also had an unusually strong PRE that was not at the start of the X-ray burst, as is expected. It is unclear whether this is a longer manifestation of the pause phenomena we see in B1 and S1, but it shows that the presence of pause-like features can depend on the energy band used.

Independently of whether the pauses are related to the

GRS $1747-312$ is a transient $\mathrm{X}$-ray source located in the core of the $9.5 \mathrm{kpc}$ distant globular cluster Terzan 6 . Given RXTE/Proportional Counter Array's $1^{\circ}$ field-of-view, in't Zand et al. (2003) could not discard the possibility that the $\mathrm{X}$-ray burst originated in a different NS-LMXB multi-peaked phenomena discussed above, our results, together with those presented by Bult et al. (2019), show that short pauses in the rise of X-ray burst light curves occur in at least two sources, suggesting that these types of pauses might be present in the X-ray light curves of Type-I bursts from other NS-LMXBs. We only know of two sources (the accreting millisecond X-ray pulsar SAX J1808.4-3658 and MAXI J1807), each with one reported X-ray burst with a clear pause during the rise. This small sample size prevents us from a detailed study on the relation between pauses and $\mathrm{X}$-ray burst characteristics/properties, and naturally it is unclear whether the pauses seen in B1 and S1 are caused by the same mechanism. However, under the assumption that we are seeing manifestations of the same phenomena in B1 and S1, even with a sample of two we can conclude that pauses in the X-ray bursts are possible in both pulsating and non-pulsating systems, suggesting that the strength of the magnetic field might not play an important role, unless the lack of pulsations in MAXI J1807 is due to an alignment between rotation and magnetic axes (Lamb et al. 2009a,b). In addition, the occurrence of the pause does not appear to depend on the composition of the burning material either, or whether the X-ray burst reaches PRE.

To our knowledge, standard X-ray burst models do not predict pauses in the X-ray burst rise. However, we note that Keek et al. (2012) finds X-ray bursts with pauses in their rise when modelling X-ray bursts that occur shortly after a superburst. In the hot, He-rich atmospheres following a superburst, X-ray bursts are initially powered by the triple$\alpha$ process. As the atmospheric temperature begins to rise due to these $\mathrm{X}$-ray bursts, $\alpha$-captures start playing a role powering the peaks of the X-ray bursts. It is during this transition that X-ray bursts with pauses in the rise occur, as it takes some time to ignite the second burning stage through $\alpha$-captures. At the moment, it is unclear whether the model of Keek et al. (2012) could be adapted to explain our results, i.e. whether an X-ray burst can show pauses independently of the occurrence of a superburst to create a hot He-rich atmosphere.

\section{ACKNOWLEDGEMENTS}

A.A. thanks M. Pahari and M. Ozbey-Arabaci for discussion on the X-ray imaging observations. A.A. also thanks the referee for making helpful suggestions that improved the paper, and thanks C. L. Greenwell and N. Wragg for general discussion and support. D.A. acknowledges support from the Royal Society. G.C.M. and D.A. acknowledge support from the Royal Society International Exchanges "The first step for High-Energy Astrophysics relations between Argentina and UK". S.R. acknowledges support from the Chinese Academy of Science (CAS) President's International Fellowship Initiative (PIFI), National Natural Science Foundation of China (NSFC) grant, and the International Postdoctoral Exchange fellowship. T.G. has been supported in part by the Scientific and Technological Research Council (TÜBITAK) 119F082, Royal Society Newton Advanced Fellowship, NAF $\backslash$ R2 $\backslash 180592$, and Turkish Republic, Directorate of Presidential Strategy and Budget project, 2016K121370. C.M. is supported by an appointment to the NASA Postdoctoral Program at the Marshall Space Flight 
Center, administered by Universities Space Research Association under contract with NASA.

\section{DATA AVAILABILITY}

This work made use of data provided by the High Energy Astrophysics Science Archive Research Center (HEASARC).

\section{REFERENCES}

Arzoumanian Z., et al., 2019, The Astronomer's Telegram, 13239 Bhattacharyya S., Strohmayer T. E., 2006a, ApJ, 636, L121 Bhattacharyya S., Strohmayer T. E., 2006b, ApJ, 641, L53

Bhattacharyya S., Strohmayer T. E., 2007, ApJ, 656, 414

Boirin L., Keek L., Méndez M., Cumming A., in't Zand J. J. M., Cottam J., Paerels F., Lewin W. H. G., 2007, A\&A, 465, 559 Bradt H. V., Rothschild R. E., Swank J. H., 1993, A\&AS, 97, 355 Bult P., et al., 2018, ApJ, 859, L1

Bult P., et al., 2019, ApJ, 885, L1

Burrows D. N., et al., 2003, in Proc. SPIE. pp 1320-1325, doi:10.1117/12.461279

Casares J., Jonker P. G., 2014, Space Sci. Rev., 183, 223

Cooper R. L., Narayan R., 2007, ApJ, 657, L29

Degenaar N., et al., 2018, Space Sci. Rev., 214, 15

Galloway D. K., Keek L., 2017, arXiv e-prints, p. arXiv:1712.06227

Galloway D. K., et al., 2008, ApJS, 179, 360

Galloway D. K., et al., 2020, ApJS, 249, 32

Gehrels N., 2004, in ESA Special Publication: 5th INTEGRAL Workshop on the INTEGRAL Universe. p. 777

Gendreau K., Arzoumanian Z., 2017, Nature Astronomy, 1, 895

HEASARC 2014, HEAsoft: Unified Release of FTOOLS and XANADU (ascl:1408.004)

Harrison F. A., et al., 2013, ApJ, 770, 103

Jahoda K., et al., 2006, ApJS, 163, 401

Jaisawal G. K., et al., 2019, ApJ, 883, 61

Jiménez-Ibarra F., et al., 2019, MNRAS, 484, 2078

Keek L., Heger A., in't Zand J. J. M., 2012, ApJ, 752, 150

Keek L., Ballantyne D. R., Kuulkers E., Strohmayer T. E., 2014, ApJ, 797, L23

Keek L., et al., 2018, ApJ, 855, L4

Klein-Wolt M., van der Klis M., 2008, ApJ, 675, 1407

Kuulkers E., et al., 2003, A\&A, 399, 663

Lamb F. K., et al., 2009a, ApJ, 705, L36

Lamb F. K., et al., 2009b, ApJ, 706, 417

Lewin W. H. G., Vacca W. D., Basinska E. M., 1984, ApJ, 277, L57

Lewin W. H. G., van Paradijs J., Taam R. E., 1993, Space Sci. Rev., 62, 223

Lyu M., Méndez M., Zhang G., Keek L., 2015, MNRAS, 454, 541

Matsuoka M., et al., 2009, PASJ, 61, 999

Maurer I., Watts A. L., 2008, MNRAS, 383, 387

Mihara T., et al., 2011, PASJ, 63, S623

Negoro H., et al., 2017, The Astronomer's Telegram, 10208

Patruno A., Watts A. L., 2012, arXiv e-prints, p. arXiv:1206.2727

Rapisarda S., et al., 2019, The Astronomer's Telegram, 13173

Schatz H., et al., 2001, Phys. Rev. Lett., 86, 3471

Shidatsu M., et al., 2017, ApJ, 850, 155

Shidatsu M., et al., 2019, The Astronomer's Telegram, 13097

Spitkovsky A., Levin Y., Ushomirsky G., 2002, ApJ, 566, 1018

Strohmayer T., Bildsten L., 2003, In Compact Stellar Xray Sources. Cambridge University Press, pp 113-156 (arXiv: astro-ph/0301544)

Strüder L., et al., 2001, A\&A, 365, L18
Tauris T. M., van den Heuvel E. P. J., 2006, In Compact Stellar X-ray Sources. Cambridge University Press, pp 623-665 (arXiv: astro-ph/0303456)

Tawara Y., et al., 1984, ApJ, 276, L41

Tetarenko A. J., et al., 2018, ApJ, 854, 125

Watts A. L., 2012, ARA\&A, 50, 609

Watts A. L., Maurer I., 2007, A\&A, 467, L33

Watts A. L., Strohmayer T. E., Markwardt C. B., 2005, ApJ, 634,547

Wilms J., Allen A., McCray R., 2000, ApJ, 542, 914

Worpel H., Galloway D. K., Price D. J., 2013, ApJ, 772, 94

Worpel H., Galloway D. K., Price D. J., 2015, ApJ, 801, 60

Zhang G., et al., 2009, MNRAS, 398, 368

in't Zand J. J. M., Strohmayer T. E., Markwardt C. B., Swank J., 2003, A\&A, 409, 659

in't Zand J. J. M., et al., 2017, A\&A, 606, A130

van Paradijs J., 1978, Nature, 274, 650

van Paradijs J., et al., 1986, MNRAS, 221, 617

van der Klis M., 2006, In Compact Stellar X-ray Sources. Cambridge University Press, pp 39-112 (arXiv:astro-ph/0410551)

This paper has been typeset from a TEX/LATEX file prepared by the author. 